\title{
Alte Verkehrswege in der modernen Kulturlandschaft - Sinn und Zweck des Inventars historischer Verkehrswege der Schweiz (IVS)
}

\section{Einleitung}

Kulturlandschaften bilden nicht stabile Systeme, sondern sind dauernden Veränderungen unterworfen. Bis um die Mitte unseres Jahrhunderts wurden die vom Menschen verursachten Veränderungen im Landschaftsbild vorwiegend positiv beurteilt, da sie mit wirtschaftlichen Verbesserungen verknüpft waren (Gewässerkorrektionen, Güterzusammenlegungen, der moderne Straßenbau). Heute spüren wir in allen Bereichen, daß der technische und wirtschaftliche Fortschritt die Umwelt und damit unsere Lebensqualität in steigendem Maße gefährdet.

Im folgenden Beitrag wird zunächst gezeigt, auf welche Weise die traditionelle Verkehrslandschaft verändert worden ist. Aus der Sorge um die Erhaltung von Wegen und Wegbegleitern als Elemente der gewachsenen Kulturlandschaft heraus ist der Auftrag des Bundesamtes für Forstwesen (BFF) entstanden, ein "Inventar historischer Verkehrswege der Schweiz» (IVS) zu erstellen. Das Inventar ist als Hinweisinventar konzipiert, das als Entscheidungshilfe für die Raumplanung dienen wird.

\section{Die Umgestaltung der Verkehrsnetze - Problemstel- lung}

Bei einer Systematisierung der Umformung von Verkehrsnetzen lassen sich folgende Unterscheidungen treffen:

\subsection{Lokale Veränderungen}

Die Verkehrslandschaft vor der Motorisierung war reich an Kleinformen und wegbegleitenden Einrichtungen. Die allmähliche Anpassung des überlieferten Wegnetzes führte zu vielen kleinen Wegkorrekturen. Hohlwege wurden ausgefüllt (Abb. 1), Stützmauern aus Bruchsteinen verschwanden unter einer Betonmauer (Abb. 2), Wegkapellen und Wegkreuze wurden abgebrochen oder versetzt (Abb.3), die Straßen erhielten Beläge. Im Zuge von Verbreiterungen mußten ehemals auf den Verkehr ausgerichtete Gebäude (Zollstätten, Wirtshäuser) weichen, und an frühere Wegverbesserungen erinnernde Inschriften verschwanden. Alle diese Veränderungen waren im einzelnen nicht wesentlich, gesamthaft aber bewirkten sie eine Verarmung und schließlich eine Zerstörung der gewachsenen Kulturlandschaft. Besonders eindrückliche Schilderungen dieses Prozesses bieten die Bilder von J.E. MÜLLER (1973) in «Alle Jahre wieder saust der Preßlufthammer nieder...».

\subsection{Bandartige Umgestaltungen in Verkehrskorridoren}

Vor dem Bau des Autobahnnetzes suchte man durch Verbreiterung der Fahrbahn oder durch Strecken von Kurven die bestehenden Straßen an die neuen Bedürfnisse anzupassen. Seit den 1960er Jahren brachte der Bau der Autobahnen den davon betroffenen Gemeinden eine völlige Umstrukturierung des Grundeigentums und eine Neugestaltung des lokalen Verkehrsnetzes. Güterzusammenlegungen waren die Voraussetzung für die geplanten Routen (AERNI \& PFISTER 1973: 223; PFISTER 1977: 27). Flurwege, die vorher sowohl der Landwirtschaft wie auch dem Fußgänger und Wanderer gedient hatten, wurden durch die neuen Großbauten unterbrochen oder verlegt (Abb.4). Ferner belasten die Großbauten die Landschaft visuell, und die Lärmimmissionen beeinträchtigen die Wohn- und Lebensqualität. Längst ist der naive Glaube verschwunden, wonach die Autobahnen sich konfliktfrei in die Landschaft einbetten lassen, wie seinerzeit der Chef des Eidgenössischen Amtes für Straßen- und Flußbau prophezeite (RUCKLI 1960: 6).

\subsection{Flächenhafte Veränderungen in Gebieten intensi- vierter Nutzung}

In den dreißiger Jahren haben viele Güterzusammenlegungen stattgefunden, wobei die Arrondierung der Höfe im Vordergrund stand. Dies änderte sich mit dem Übergang zu den Gesamt- oder Integralmeliorationen, welche die gesamte Landschaft erfaßten. Die bis dahin verbliebenen Feuchtgebiete wurden entwässert, Bäche eingedolt, Hecken und Einzelbäume entfernt. Die Parzellen erhielten Rechteckform. Die

K. Aerni, Prof., u. Hp. Schneider, Geographisches Institut der Universität Bern, Hallerstrasse 12, 3012 Bern 


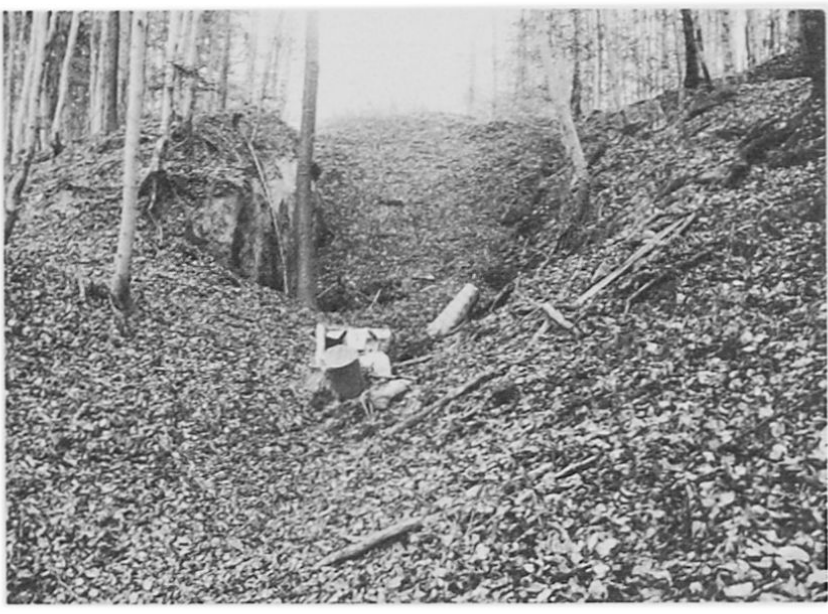

Abb. 1 Boll (BE): Ehemaliger Hohlweg mit Schutt aufgefüllt. Standort: $608650 / 200600$; Bildmitte: Az: 700 A o/o0 LK: 1167.

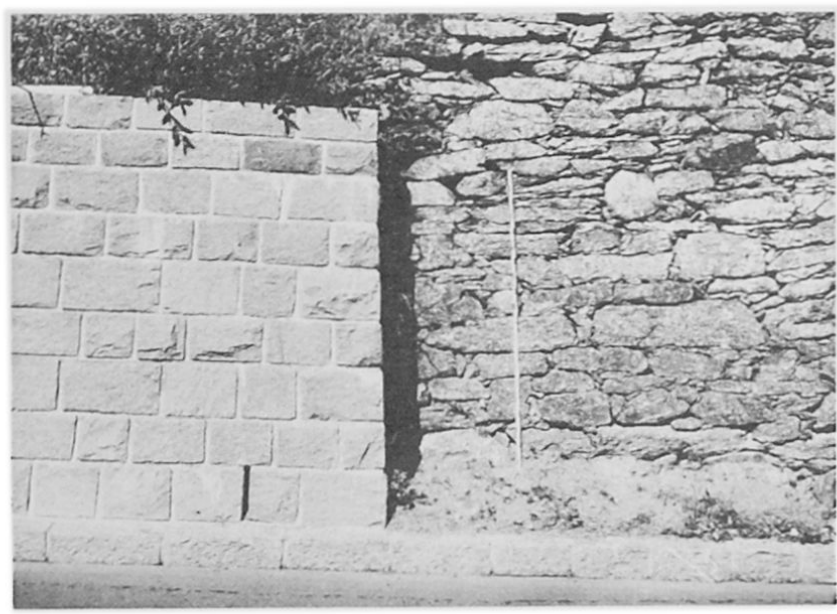

Abb. 2 Simplon (VS): Zwei Generationen von Stützmauern (rechts aus der napoleonischen, links aus der heutigen Zeit). Standort: $648800 / 115000 / 1230 \mathrm{~m}$; Bildmitte: Az: 5500 A o/oo; LK: 1309.

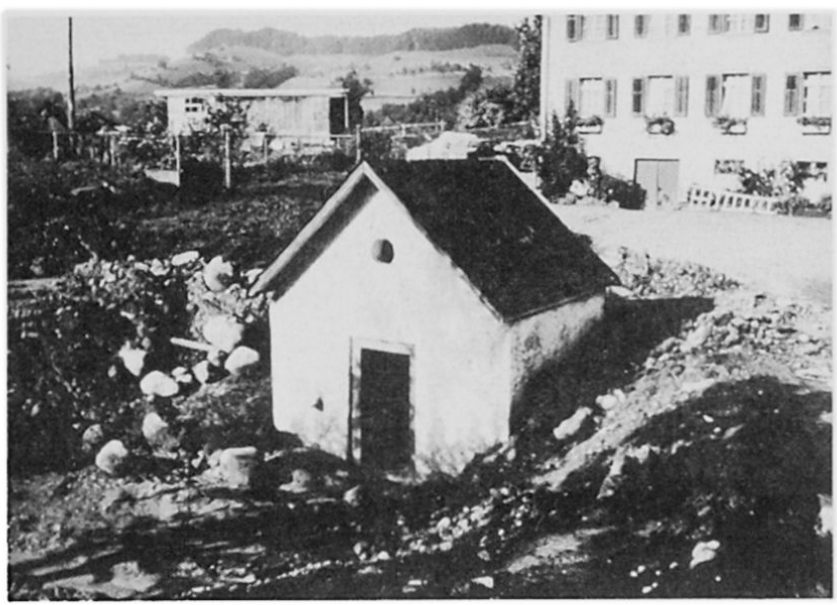

Abb. 3 Wolhusen (LU): Wegkapelle kurz vor dem Abbruch. Standort: 648 400/207 425; Bildmitte: Az: 200 A o/oo; LK: 1149.

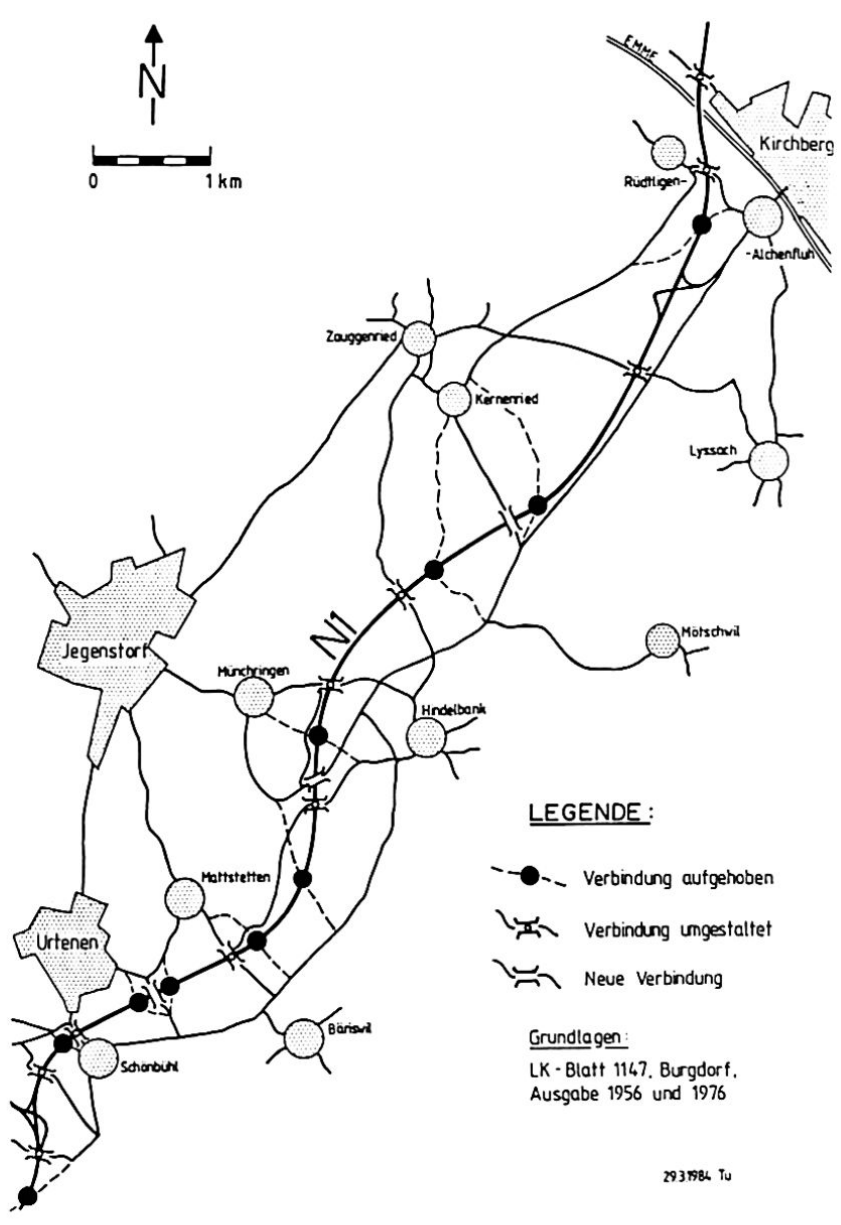

Abb. 4 Unterbrüche im lokalen Wegnetz durch den Bau der "N1" im Abschnitt Schönbühl-Kirchberg.

durch jahrhundertelangen Feldbau gewachsenen morphologischen Kleinformen wie Ackerraine und Hohlwege verschwanden. Die Kulturlandschaft wurde ausgeräumt und erhielt ein neues, rechtwinkliges Wegsystem, heute oft mit einem Hartbelag versehen (vgl. EWALD 1978). Diese Landschaften haben durch die Technik ein neues Gesicht erhalten und haben dabei weitgehend ihren Wert als Erholungsgebiet eingebüßt (Abb. 5, 6).

Ähnliche Tendenzen zeichnen sich heute in vielen Waldgebieten ab. Bis in unsere Zeit hinein sind in den Wäldern wesentlich mehr alte Wegspuren erhalten geblieben als im offenen Kulturland. Dies scheint sich nun durch den forcierten Bau von modernen Forststraßen zu ändern (Abb.7). Die Umstellung auf Spezialfahrzeuge zum Holzschlag und Abtransport erfordert die Befahrbarkeit des Waldes und veranlaßt damit die Nivellierung von Hohlwegen. Da die neuen Walderschließungsstraßen häufig hangparallel mit möglichst geringer Steigung angelegt werden, schneiden und zerstören sie oft auch die in der Fallinie angelegten historischen Verkehrswege. Damit droht eine Gleichförmigkeit der Waldwege, die ihr Pendant im Ersetzen des Plenterwaldes durch reine Nadelwaldplantagen hat. 


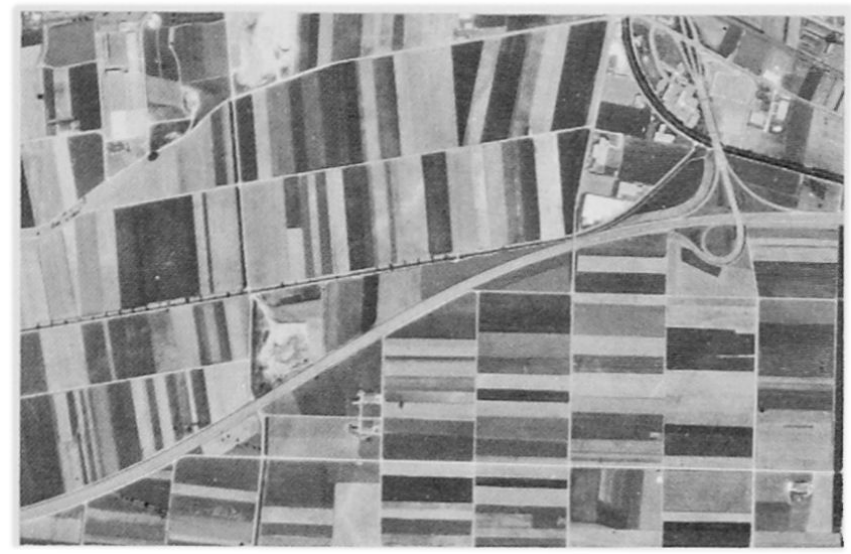

Abb. 5 Oensingen (SO): Meliorierte, schachbrettförmige Landschaft im Raum Autobahnanschluss.

Bildmitte: $621500 / 236300 / 455 \mathrm{~m}$; Senkrechtaufnahme. LK: 1108. Aufnahme des Bundesamtes für Landestopographie Nr. 9801 vom 18.6.1975, Flugl. 16. Reproduziert mit Bewilligung des Bundesamtes für Landestopographie vom 2.11.1983.

\subsection{Rückentwicklung von Verkehrsachsen}

Neben den Räumen mit wachsendem Verkehr gibt es auch Passivräume, in welchen einst wichtige Verbindungen verlassen wurden und heute nur noch als fossile Wegspuren erhalten sind. Dies betrifft vor allem die meisten alpinen Übergänge, deren Bedeutung sich seit dem letzten Jahrhundert auf die wenigen ausgebauten Pässe verlagert hat. Die Spuren dieser Wege, deren Anfänge beim Großen St.Bernhard und den Bündnerpässen in die gallorömische Zeit zurückreichen, haben sich recht gut bis in die Mitte unseres Jahrhunderts erhalten. Seither sind diese Anlagen, vor allem im zentralalpinen Bereich, über weite Strecken durch Kraftwerkbauten, ForststraBen, touristische und militärische Neuanlagen sowie besonders durch Kabelverlegungen zerstört worden (AERNI 1961, 1971, 1975) (Abb. 8).

Ähnlich wie in den Alpen haben auch im Jura verschiedene einst wichtige Übergänge durch den Bau der Talstraßen in den Klusen ihre Bedeutung verloren. Beispielsweise existierte noch 1873 zwischen Champoz $(589280 / 234200)$ und Les Ecorcheresses (587950/236 100) eine Fahrstraße, an deren Stelle heute nicht einmal mehr ein zusammenhängender Fußweg vorhanden ist.

Für das Mittelland sei auf die Höhenstraße des Wägesse hingewiesen, der noch im 17. Jahrhundert eine wichtige Verbindung zwischen dem Emmental und der Stadt Bern darstellte (PROBST 1981).

\subsection{Folgerungen}

Zusammenfassend stellen wir fest, daß in den letzten Jahrzehnten durch die Tätigkeit privater wie öffentlicher Institutionen viele Teile der Naturlandschaft und der unsere Geschichte widerspiegelnden Kulturland-

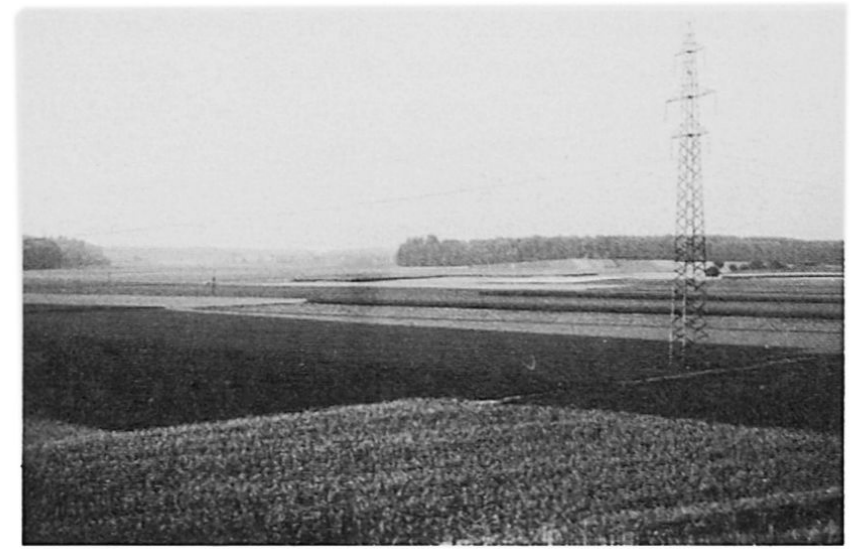

Abb. 6 Ersigen (BE): Langweilige, "ausgeräumte» Landschaft Standort: 611 750/217 520; Bildmitte: Az: 5900 A o/oo; LK 1147 .

\begin{tabular}{|c|c|c|c|}
\hline Baujahr & & $\begin{array}{l}\text { / Ausbau } \\
\mathrm{km}\end{array}$ & $\begin{array}{l}\text { Aufwand } \\
\text { in Mlo } \mathrm{Fr} \text {. }\end{array}$ \\
\hline $1965-74$ & \multirow[t]{7}{*}{ Mittelwert } & 454 & --- \\
\hline 1975 & & 425 & 70,0 \\
\hline 1976 & & 617 & 88,6 \\
\hline 1977 & & 440 & 74,3 \\
\hline 1978 & & 361 & 59,1 \\
\hline 1979 & & 424 & 73,8 \\
\hline \multirow[t]{3}{*}{1980} & & 323 & 47,2 \\
\hline & Total 1965-8 & 7135 . & \\
\hline & Total 1975-8 & & 413,0 \\
\hline
\end{tabular}

Quelle: Geschaftsber1chte OFI 1975, 1976, 1977, 1978, 1979, 1980

Abb. 7 Waldstrassenbau in der Schweiz 1965-1980.

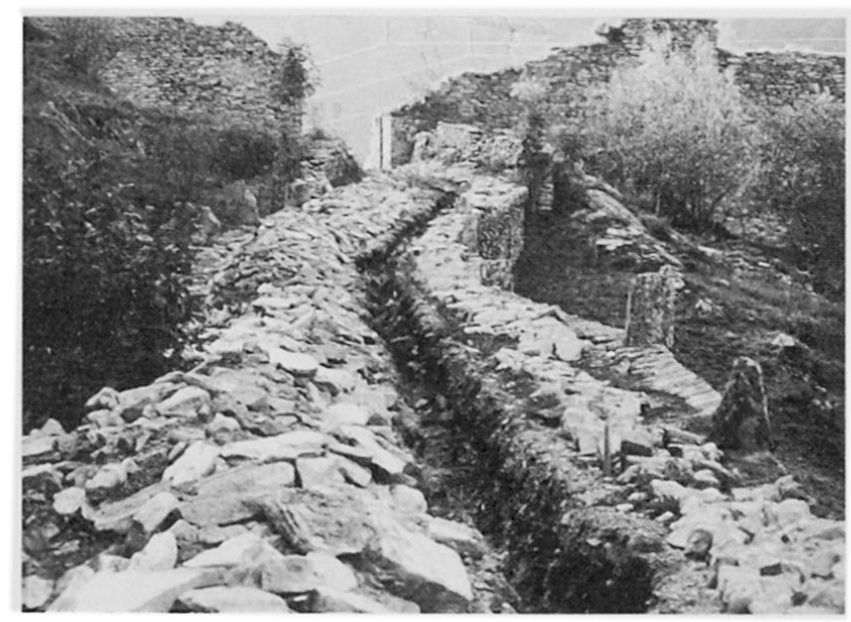

Abb. 8 Promontogno, Sperre Castelmur (GR): Die Plästerung eines bedeutenden historischen Weges wird zur Verlegung eines Leitungskabels aufgebrochen.

Standort: 763 500/134 200/900 m; Bildmitte: Az: 4600 A o/oo; LK: 1276. 
schaft unwiederbringlich verändert worden sind. Von diesen Veränderungen wurden vor allem die aus der Zeit vor der Motorisierung stammenden Wege und Wegbegleiter betroffen, die in ihrer Anlage und individuellen Ausprägung einen wesentlichen Bestandteil unserer Erholungslandschaften darstellen. Mit der Zerstörung der Kleinobjekte und fossilen Geländespuren brechen wir den Dialog mit unserer Geschichte und mit unserer Überlieferung für uns und für unsere Nachfahren ab. Der Landschaft drohen Verarmung und zunehmende Gleichförmigkeit. Für uns sind wohl der Verlust der Identität und die Verschlechterung der Umweltqualität die schwerwiegendste Folge.

So stellt sich denn die Frage, wie Wege und Wegbegleiter als Elemente der gewachsenen Kulturlandschaft geschützt werden könnten. An sich verfügen Natur- und Heimatschutz über eine lange Erfahrung für den Schutz von Einzelobjekten (z. B. Findlinge, Bäume, Biotope, Gebäude), Landschaften (siehe KLN-Inventar $1979^{2}$ und BLN-Inventar 1977) und Siedlungen (ISOS-Inventar der schützenswerten Ortsbilder der Schweiz). Die zahlreichen Rechtsgrundlagen zum Schutz kulturgeschichtlich wertvoller Landschaften (SCHWARZE 1983: 3-4) gestatten auch den Einbezug der alten Wege in Schutzzonen (vgl. RPG Art. 17, Absatz 1, c). In den Erläuterungen zum Bundesgesetz über die Raumplanung (EJP 1981: 230) wird festgehalten, daß zu den in Schutzzonen $\mathrm{zu}$ integrierenden «Kulturdenkmälern» «geschichtlich hervorragende handwerkliche oder bauliche Leistungen vergangener Zeiten" zu zählen sind, wobei $\mathrm{Paßstraßen} \mathrm{und} \mathrm{Brücken} \mathrm{ausdrücklich} \mathrm{erwähnt} \mathrm{wer-}$ den. Bis in die jüngste Zeit hinein fehlte es aber an der Einsicht, daß die eher bandartigen Objekte der historischen Verkehrswege den logischen Zusammenhang zwischen bisher geschützten oder noch $\mathrm{zu}$ schützenden Einzelobjekten herstellen und in diesem Sinne schützenswert sind.

\section{Der Auftrag des Bundesamtes für Forstwesen (BFF) zur Erarbeitung eines Inventars historischer Verkehrs- wege der Schweiz (IVS)}

Um die Gefahr der weiteren Zerstörung historischer Verkehrswege und mit ihnen in Beziehung stehender Objekte zu reduzieren, hat sich das BFF 1980 entschlossen, ein Inventar der historischen Verkehrswege der Schweiz (IVS) erstellen zu lassen. Den gesetzlichen Rahmen bildet das Bundesgesetz über den Natur- und Heimatschutz (NHG, 451) vom 1. Juli 1966. Der Bund ist demnach besorgt, das heimatliche Landschafts- und Ortsbild, die geschichtlichen Stätten sowie die Natur- und Kulturdenkmäler des Landes zu schonen, die Kantone in der Erfüllung ihrer Aufgaben des Natur- und Heimatschutzes zu unterstützen und die Zusammenarbeit mit ihnen zu sichern (Art. 1, a und b).

Zur Unterscheidung der Schutzwürdigkeit von Kulturobjekten (Art.4) läßt der Bund Inventare von Objekten von nationaler Bedeutung erstellen (Art. 5). Er stellt Grundsätze auf für die Beurteilung von schützenswerten Objekten (Art.6-8) und regelt schließlich die Beiträge zur Unterstützung der Naturund Heimatschutzbestrebungen von Kantonen, Gemeinden und privaten Institutionen (Art. 13-15).

\section{Ziele}

\subsection{Entscheidungsgrundlagen für die Raumplanung}

Gemäß Auftrag liegt das Hauptziel unserer Arbeit darin, ein Hinweisinventar als Entscheidungshilfe für die Raumplanung zu erstellen.

Nach dem Bundesgesetz über die Raumplanung (RPG, 700) vom 22. Juni 1979 sind die Kantone verpflichtet, Richt- und Nutzungspläne zu erstellen (RPG 700, Art. 35). In den für die Behörden verbindlichen Richtplänen (Art. 9) muß unter anderem festgelegt werden, welche Gebiete «besonders schön, wertvoll, für die Erholung oder als natürliche Lebensgrundlage bedeutsam sind» (Art. 6, Abs. 2b).

Die Belange von Natur- und Heimatschutz können beim Erstellen von Richtplänen aber nur dann Berücksichtigung finden, wenn ihre Ansprüche auch klar umrissen sind. Hinweisinventare wie das IVS sollen somit erste wichtige Entscheidungsgrundlagen für die integrale Planung bilden.

\subsection{Lösungsvorschläge für eine integrale Planung}

Über die Inventarisierung hinaus ist ein weiteres wichtiges Ziel des IVS, den Raumplanern konkrete Lösungsvorschläge im Sinne einer Erhaltung, einer Weiterverwendung oder auch einer Wiederherstellung von schützenswerten historischen Wegobjekten vorzulegen. Anzustreben ist diesbezüglich eine möglichst weitgehende Integration in die Richt- und Nutzungspläne, besonders in den Bereichen der Erholungslandschaftsplanung.

Grundsätzlich sind Relikte historischer Verkehrswege, ihrer Bedeutung entsprechend, möglichst umfassend zu schützen. Allerdings ist Schutz nur eine Funktion, die sich in der Regel einer andern Funktion überlagert (GROSJEAN 1982: 122).

Es gilt hier zu berücksichtigen, daß die heutige Kulturlandschaft ein Spannungsfeld unterschiedlichster Interessen darstellt. Es entstehen Nutzungsüberlagerungen, die wiederum in die integrale Planung einzubeziehen sind. Den Idealfall finden wir dort, wo sich gleiche oder zumindest ähnliche Nutzungsansprüche überlagern. 


\subsection{1 Überlegungen beim Vorliegen ähnlicher oder gleicher Nutzungsansprüche}

\section{Zur Nutzung der Wanderwege}

Sehr ähnliche Nutzungsansprüche liegen etwa dort vor, wo sich die Interessen für eine Kulturlandschaftserhaltung mit den Interessen der Erholungslandschaftsplanung kombinieren lassen.

So dürften die ausgeschiedenen schützenswerten historischen Wegobjekte in Zukunft eine wichtige Grundlage bilden beim Aufstellen von Wanderwegkonzepten oder auch für spezifische Bereiche der Landschaftsplanung.

\section{Zur Nutzung der Forst- und Flunwege}

Schützenswerte Objekte historischer Verkehrswege können durchaus in die Erschließung von Forst- und Landwirtschaftsgebieten einbezogen werden. Abzuklären ist in diesem Zusammenhang aber vor allem die Verträglichkeit.

Von seiten des Kulturlandschaftsschutzes und im speziellen des Inventars historischer Verkehrswege der Schweiz (IVS) sind unbedingt Nutzungsvorschriften (Beispiel: partielles Fahrverbot, Fahrverbot für schwere Lastwagen usw.) sowie Ausbauverbote für die entsprechenden Wege im Bereich von schützenswerten Objekten erforderlich.

\subsection{2 Überlegungen beim Auftreten von Nutzungskonflikten}

Der moderne Straßenbau, die Großmeliorationen und Güterzusammenlegungen bilden die bedeutendsten Eingriffe in die ländliche Kulturlandschaft. Daraus entstehen häufig Konflikte, die bisher oft diskussionslos zugunsten der Neubauten entschieden worden sind.

Wichtig ist der Grundsatz, daß kulturlandschaftliche Ansprüche anzumelden sind, bevor neue Richtpläne erstellt und Projekte sanktioniert sind.

Nötigenfalls müssen Kompromiß- und Alternativvorschläge gemacht werden!

\subsection{Orientierung der Öffentlichkeit}

Inventare des Natur- und Heimatschutzes sind Hinweisinventare und nicht rechtsverbindlich. Sie sind deshalb auf die Unterstützung durch Behörden und Planungsgremien sowie der Öffentlichkeit angewiesen.

Es ist daher wesentlich, daß die Öffentlichkeit frühzeitig darauf aufmerksam gemacht wird, daß durch den Schutz alter Wege zwei wesentliche Anliegen unserer Zeit erfüllt werden können. Einmal geht es um Schutz wertvoller Landschaften und Kulturdenkmäler, zum andern um Erhaltung und maßvolle Weitergestaltung der Erholungslandschaften. Die Orientierung der Öffentlichkeit hat in zwei Schritten zu erfolgen:

Eine Orientierung über den eigentlichen Gegenstand des Inventars soll zunächst die Bevölkerung und die
Behörden auf den schützenswerten Charakter historischer Wege aufmerksam machen. Bereits die Kenntnis über das Vorhandensein schützenswerter Objekte bewirkt in an sich interessierten Kreisen einen ersten Schutz. So hat man z. B. in Deutschland bei ähnlichen Inventarisationen festgestellt, da $\beta$ des öftern Kulturobjekte nicht mutwillig, sondern aus Unkenntnis beschädigt oder gar vernichtet werden (GEBESSLER/EBERL 1980: 64). Auch beim ISOS (Inventar der schützenswerten Ortsbilder der Schweiz) zeigt sich, $\mathrm{da} \beta$ die vorhandenen Inventarteile bereits vor dem Abschluß des Gesamtwerkes konsultiert werden.

In einem zweiten Schritt sind bei der Aufnahme des Inventars und bei Ausgestaltung der Vorschläge lokale und regionale Organisationen zu orientieren und einzubeziehen, damit das allgemeine Interesse an Landschafts- und Ortsbild, geschichtlichen Stätten sowie Natur- und Kulturdenkmälern von breiten Kreisen getragen werden kann.

\subsection{Anschluß an bestehende Inventare}

Mit den umfassenden Inventarisationen des Denkmalschutzes und der Denkmalpflege sowie vor allem dem ISOS hat man in bezug auf die Aufnahme und den Schutz traditioneller Kulturlandschaftselemente schon große Erfahrungen gesammelt. Es ist deshalb von Bedeutung, hier sowohl den methodischen (Vorgehen) wie auch den praktischen (Schutzmöglichkeiten) Anschluß für unsere neue Aufgabe zu suchen, um letztlich auch die Vergleichbarkeit der Resultate zu ermöglichen.

Wie bei den laufenden Kulturlandschaftsinventarisationen geht es auch beim Inventar historischer Verkehrswege der Schweiz (IVS) in erster Linie um die Aufnahme und den Schutz von Einzelobjekten. Im Sinne einer Rekonstruktion ganzer historischer Wegachsen bietet sich uns nun aber die Möglichkeit, bisher isolierte, unabhängig voneinander aufgenommene Kulturobjekte, Kulturdenkmäler und Ortschaften in einen funktionalen Zusammenhang zu bringen. Dieser thematisch sehr engen Wechselbeziehung zwischen den verschiedenen Kulturlandschaftsinventaren wird heute in der Praxis, das heißt in der Konfrontation mit der aktuellen Orts-, Regional- und Landesplanung eigentlich wenig Bedeutung beigemessen. Eine bessere Zusammenarbeit müßte erreichen, da $\beta$ die traditionelle Kulturlandschaft als ein Ganzes und nicht als ein heterogenes Gebilde unabhängiger Einzelelemente betrachtet wird. Folgendes Beispiel soll die Wichtigkeit dieser Feststellung unterstreichen: Eine Wallfahrtskapelle und ein alter Pilgerweg besitzen durch ihre funktionale Beziehung zusammen wesentlich mehr Gewicht und folglich auch die größeren Chancen, im Rahmen einer integralen Planung als Elemente der traditionellen Kulturlandschaft entsprechend bewertet zu werden. Zusätzlich würde hier eine Nichtberücksichtigung des einen 
Kulturelementes automatisch auch eine Abwertung des andern mit sich ziehen.

\section{Forschungskonzept}

Die Hauptaufgabe einer Inventarisation liegt in der systematischen Aufnahme und einer möglichst fundierten wissenschaftlichen Untersuchung der einzelnen Kulturlandschaftselemente.

Da unsere Kulturlandschaft einem ständigen Wandel unterworfen ist, stehen die Arbeiten am IVS unter hohem zeitlichem Druck. Um einerseits planmäßige Arbeiten zu ermöglichen und andererseits auf kurzfristig auftretende Konflikte reagieren zu können, sehen wir nach unserem Gesamtarbeitskonzept (Abb. 9) eine Zweiteilung vor: Das mittelfristige Konzept (wissenschaftliche Inventarisierung) zielt auf ein systematisches Inventar, das kurzfristige Konzept löst aktuelle Konflikte und liefert Teilergebnisse zuhanden des Inventars.

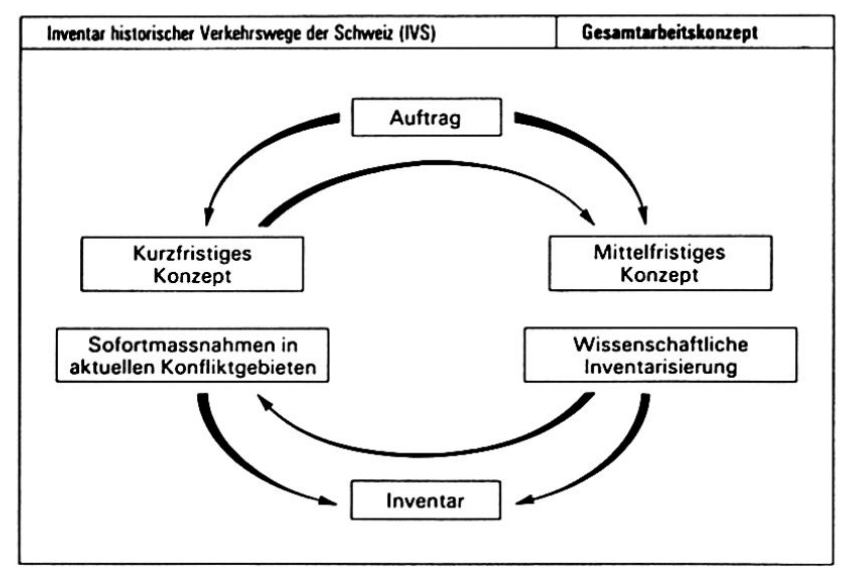

Abb. 9 Gesamtarbeitskonzept.

\subsection{Mittelfristiges Konzept (Abb. 10)}

Die wissenschaftliche Inventarisation nach mittelfristigem Konzept läßt sich in 4 Phasen gliedern:

Phase $l$ entspricht der eigentlichen Vorbereitung. Die wichtigste Grundlage bildet hier die bereits publizierte, über die ganze Schweiz erstellte Bibliographie IVS 1982 (IVS 1983). - Sie umfaßt eine Zusammenstellung der veröffentlichten Literatur und ist nach Kantonen und Verkehrsleitlinien gegliedert. Zusätzlich enthält die Bibliographie erste Hinweise auf die Entwicklung und den Verlauf von Verbindungen sowie Hinweise auf sogenannte Wegbegleiter wie Kapellen (Abb. 11), Stundensteine (Abb. 12), Zollstationen, Brücken usw.

Auf einer Übersichtskarte im Maßstab 1:300000 (Beilage zur Bibliographie, siehe auch BARRAUD/HER-

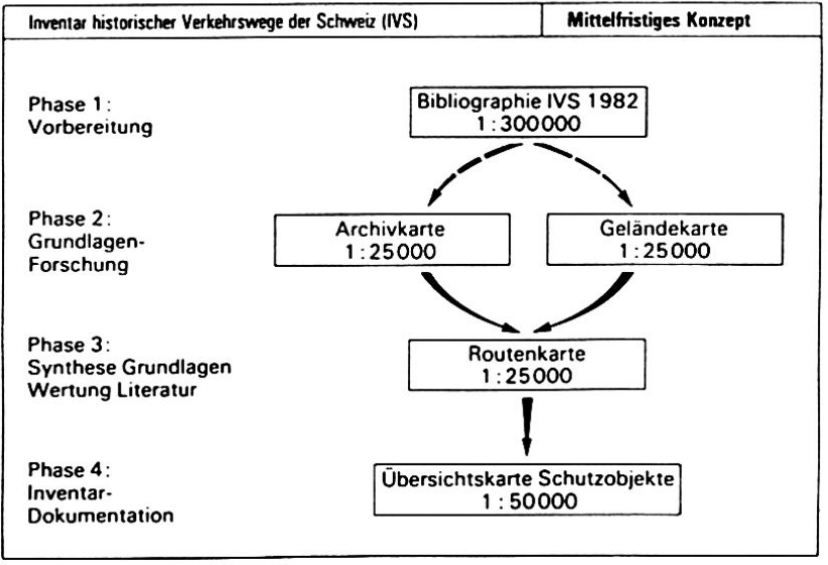

Abb.10 Mittelfristiges Konzept.

ZIG 1983) sind die verschiedenen Verkehrsleitlinien dargestellt und entsprechend den Literaturgruppen numeriert. Sie beschreiben keinen genauen Wegverlauf, sondern sind stark generalisierte Linien der Verkehrsentwicklung.

Die Bibliographie IVS 1982 soll sowohl den Bearbeitern des Inventars wie auch den Behörden und Planern ermöglichen, einen schnellen Einstieg in die Verkehrsgeschichte einer bestimmten Verbindung oder einer Region zu finden.

Die rund 900 Seiten umfassende Gesamtaufgabe der Bibliographie IVS 1982 liegt heute auf bei Bundesstellen, sämtlichen kantonalen Planungsstellen, Staatsarchiven, wichtigsten Bibliotheken sowie bei größeren «Interessengruppen».

Daneben wurde eine sogenannte Kantonsausgabe der Bibliographie IVS 1982 an Regionalplanungsstellen, kantonale Stellen für Archäologie, Denkmalschutz, Landschaftsschutz, Natur- und Heimatschutz usw. verteilt.

In Phase 2 beginnt die eigentliche Grundlagenforschung. Auf zwei getrennten Karten im Maßstab $1: 25000$ werden hier nun die Informationen einerseits aus dem Gelände und andererseits aus dem Archiv zur Darstellung gebracht.

Die Geländekarte setzt sich zusammen aus noch sichtbaren Elementen, respektive Abschnitten alter Wege. Zur Differenzierung der Wegeigenschaften unterscheiden wir zwischen der Wegoberfläche (Pflästerung [Abb. 13], anstehendes Gestein [Abb. 14], Lockermaterial [Abb. 15] usw.), dem Wegkörper (Hohlweg [Abb. 16], Hangweg [Abb. 17], Damm [Abb. 18] usw.) sowie möglichen Reliktformen ehemaliger Wege (Baumreihen [Abb. 19], Hangkante [Abb. 20] usw.). Die Geländekarte entsteht durch eine systematische und flächendeckende Begehung im Felde.

Die Archivkarte andererseits enthält möglichst objektive Informationen (keine Sekundärliteratur!) über die Verkehrsgeschichte und im speziellen über die genaue Linienführung alter Verbindungen. 


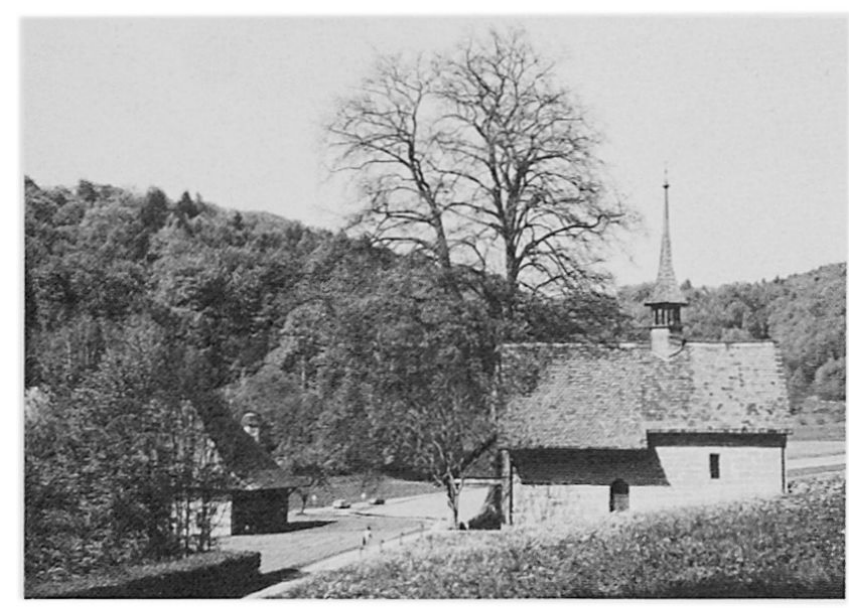

Abb.11 Burgdorf-Sommerhaus (BE): Wegkapelle und Siechenhaus.

Standort: 614 780/212 330; Bildmitte: Az: 1000 A o/oo; LK: 1147.

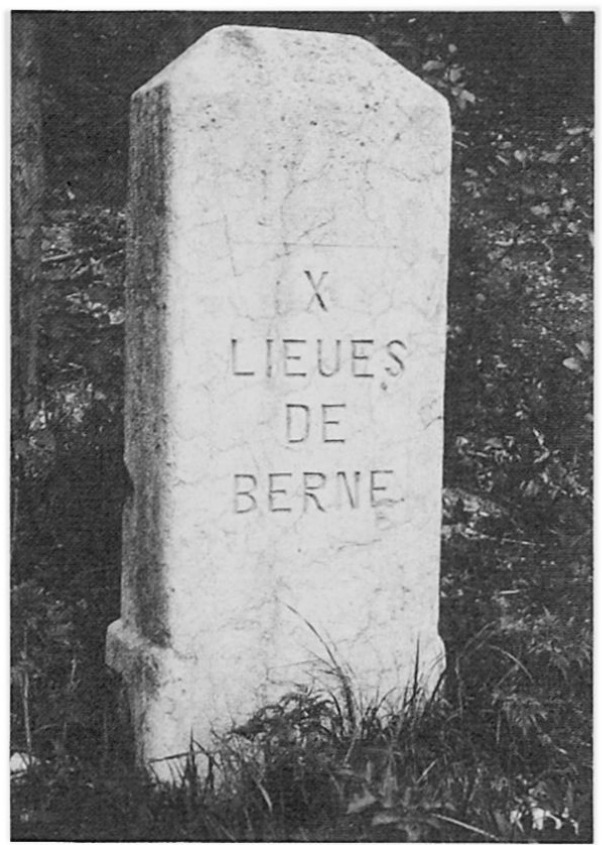

Abb.12 Sonceboz (BE): Stundenstein zeigt die Anzahl Fusswegstunden (franz. lieues) vom bernischen Zytglogge bis zum Standort des Stundensteins (1 Fusswegstunde $=4,8 \mathrm{~km}$ ). Standort: 580 700/227 575; Bildmitte: Az: 1200 A o/oo; LK: 1125.

Den Hauptinhalt der Archivkarte bilden schriftliche Quellen über Wege und wegbegleitende Objekte, Informationen aus alten Karten und Plänen, Hinweise aus bestehenden Inventaren (z. B. Archäologisches Hinweisinventar) und neueren Kartenwerken (z.B. Burgenkarte der Schweiz) sowie Angaben über Flurund Ortsnamen.

In Phase 3 entsteht mit der sogenannten Routenkarte eine Synthese aus den beiden Grundlagenkarten sowie aus der Wertung der Bibliographie.
Die Routenkarte bildet den Abschluß der wissenschaftlichen Bearbeitung und die Grundlage für die abschließende Beurteilung und Wertung der historischen Wegobjekte.

In Phase 4 erhalten wir mit der Übersichtskarte Schutzobjekte im Maßstab 1:50000 Aufschluß über die Verteilung von schützenswerten Objekten sowie über deren Art und Bedeutung (national, regional, lokal). Und schließlich bildet eine Dokumentation über das Einzelobjekt, bestehend aus einem Kurzbeschrieb mit den wichtigsten Informationen über das Objekt, einer Fotografie, einem Bewertungsblatt und einem Detailplan den Abschluß der Inventarisationsarbeiten.

\subsection{Kurzfristiges Konzept (Abb. 21)}

Das kurzfristige Konzept soll uns mit Hilfe von Sofortmaßnahmen in aktuellen Konfliktgebieten gestatten, eine Stellungnahme zu gefährdeten historischen Wegobjekten abzugeben, auch wenn sie noch nicht inventarisiert sind.

In der Regel handelt es sich hier um Konfliktsituationen zwischen der Kulturlandschaftserhaltung auf der einen und der aktuellen Planung auf der andern Seite. In Phase 1 führt diese Situation zur Ausscheidung von Gebieten zur dringlichen Bearbeitung. Nach unserer Erfahrung kann das kurzfristige Konzept beim IVS auf drei verschiedene Arten zur Anwendung kommen:

- Durch Vorziehen von möglichen Konfliktgebieten (Gebiete, in denen größere Bauprojekte bevorstehen).

- Durch Liefern von Grundlagen für Sachplanungen. Dies bringt uns eine direkte Zusammenarbeit etwa mit der Regionalplanung beim Aufstellen von Richtplänen oder mit Wanderwegvereinigungen beim Entwickeln von Wanderwegkonzepten.

- Durch «Feuerwehrübungen» bei Konflikten zwischen historischen, schützenswerten Wegobjekten und der aktuellen Planung.

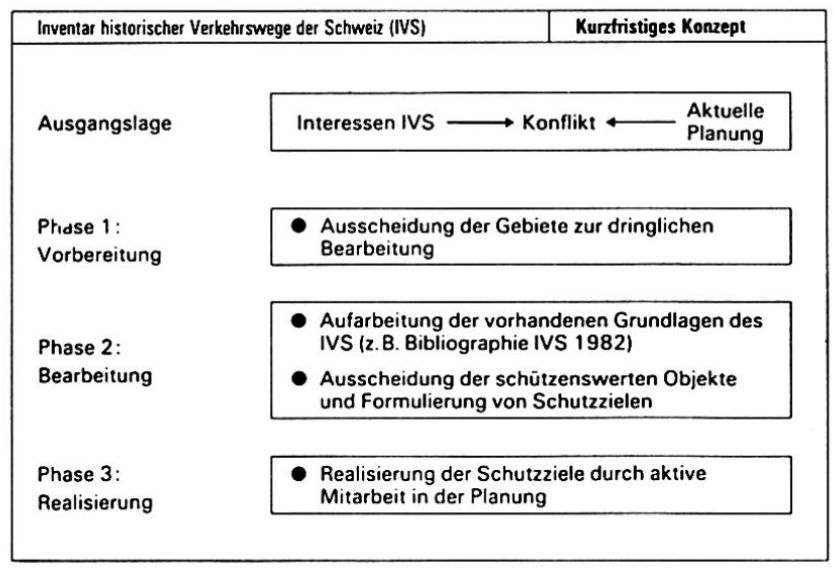

Abb. 21 Kurzfristiges Konzept. 


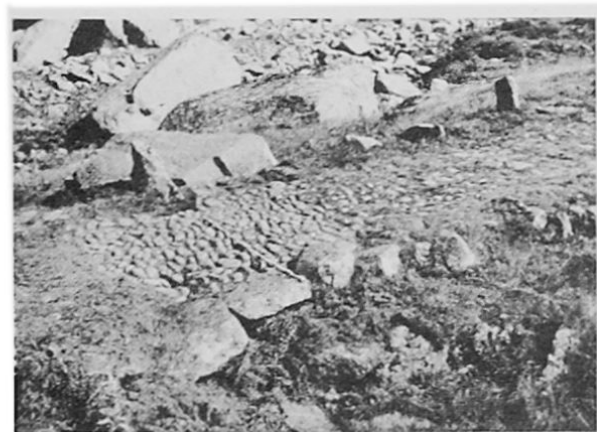

Abb.13 Gotthard-Nordseite, Mätteliboden südlich Hospenthal (UR): Kopfsteinpflaster, vor 1820.

Standort: $685875 / 160175 / 1790 \mathrm{~m}$; Bildmitte: Az: 2000 A o/oo; LK: 1231.

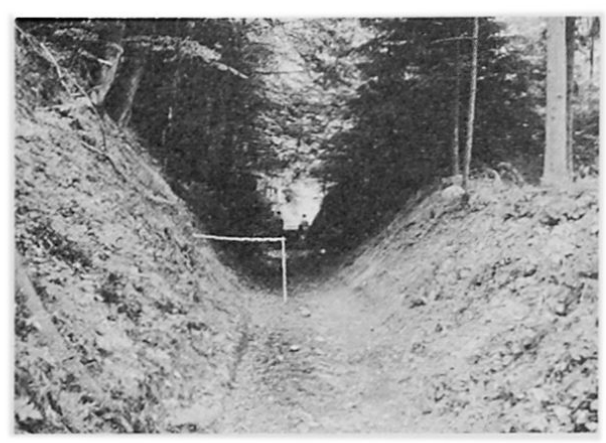

Abb. 15 Burgdorf-Hub (BE): Hohlweg im Lockermaterial. Standort: 616 320/213130; Bildmitte: Az: 4100 A o/oo; LK: 1147.

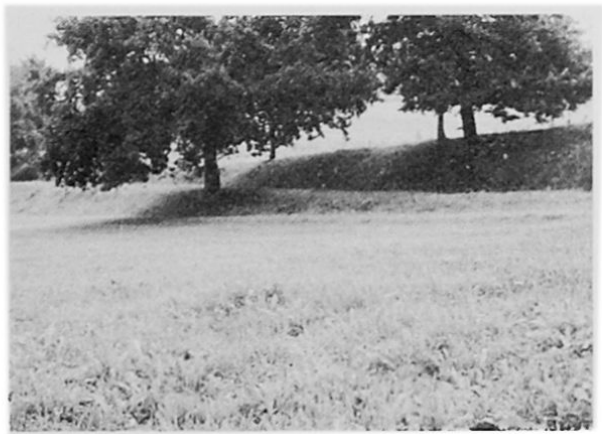

Abb. 17 Kirchberg (BE): Hangweg.

Standort: 611660/214 730; Bildmitte: Az: 1800 A o/oo; LK: 1147.

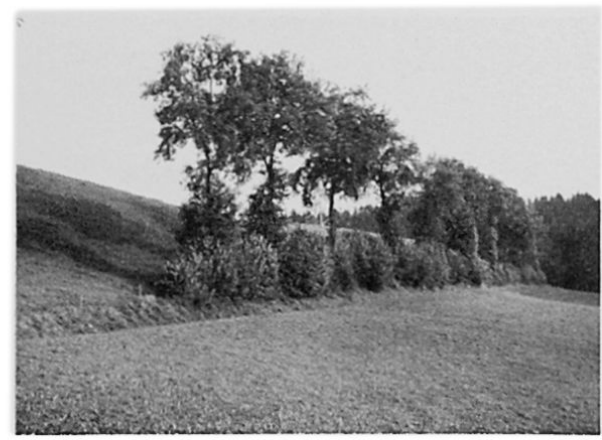

Abb.19 Diemerswil-Widlismad (BE): Auffällige Baumreihe. Standort: 598 220/206 900; Bildmitte: Az: 6200 A o/oo; LK: 1146.

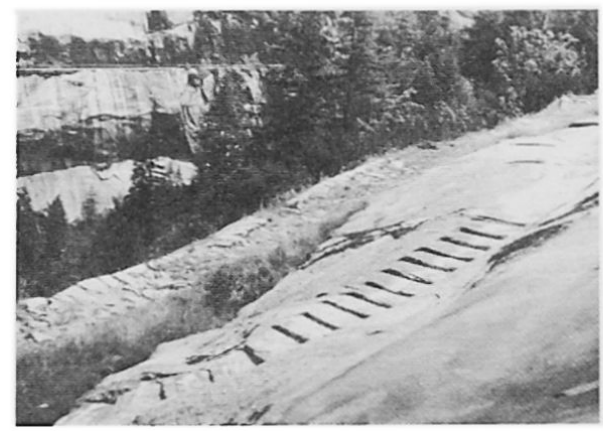

Abb.14 Grimsel-Nordseite, Hälenplatte zwischen Handegg und Kurzentännlen (BE): Stufen im anstehenden Fels.

Standort: 667 090/161 650/1510 m; Bildmitte: AZ: 1600 A o/oo; LK: 1230.

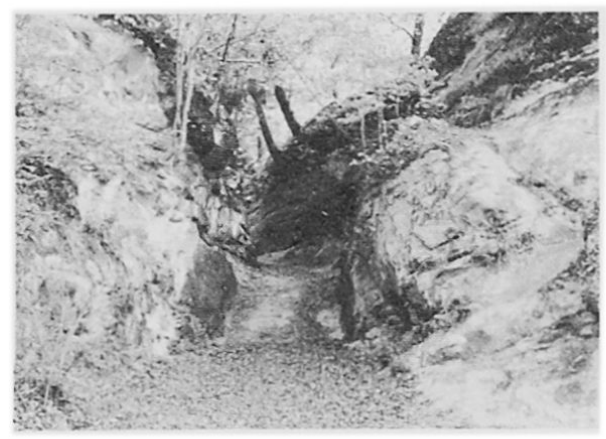

Abb. 16 Waldegg, Burgdorf (BE): Hohlweg im Sandstein.

Standort 614 660/211 280; Bildmitte: Az: 2200 A o/oo; LK: 1147.

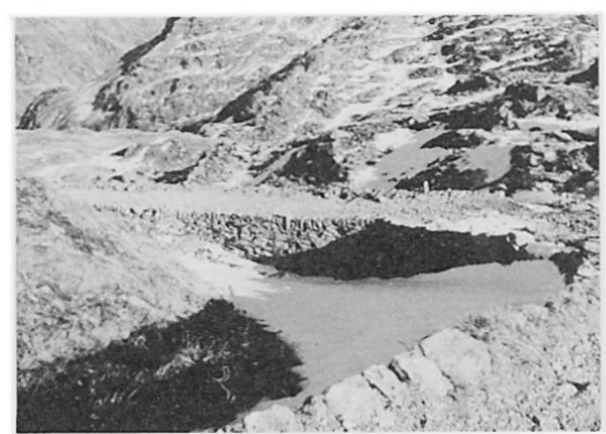

Abb. 18 Susten (BE): Dammweg.

Standort: 677 280/175 770; Bildmitte: Az: 1800 A o/oo; LK: 1211.

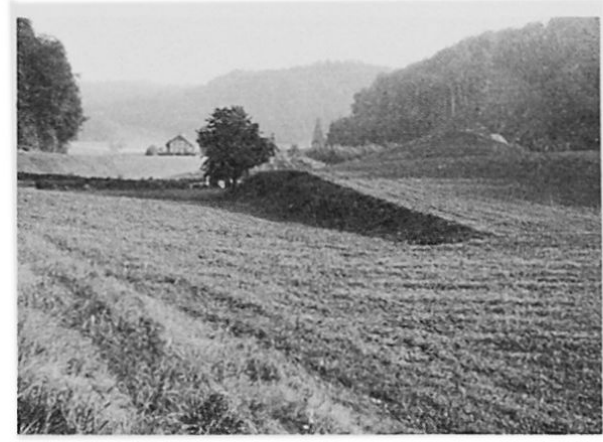

Abb. 20 Burgdorf-Sommerhaus (BE): Terrasse.

Standort 615 120/213 020; Bildmitte Az: 900 A o/oo; LK: 1147. 
In Phase 2, der Bearbeitung, geht es in der Regel darum, in möglichst kurzer Zeit ein Optimum an Informationen bereitzustellen, um schließlich eine Ausscheidung von möglichen schützenswerten $\mathrm{Ob}$ jekten sowie eine Formulierung entsprechender Schutzziele zu gewährleisten. Eine wichtige Funktion kommt in dieser Phase der bereits erwähnten Bibliographie IVS $1982 \mathrm{zu}$.

In Phase 3 schließlich sollen die anzustrebenden Schutzziele durch aktive Mitarbeit in der Planung realisiert werden.

\section{Stand der Arbeiten}

Die methodischen Vorbereitungen zum Inventar historischer Verkehrswege der Schweiz (IVS) wurden 1980 aufgenommen und im Herbst 1983 abgeschlossen. Als Testgebiet diente uns ein Ausschnitt aus dem Mittelland (LK 1: 50 000, Nr. 233 Solothurn). Daneben lieferten Auftragsarbeiten in Räumen mit unterschiedlichen geographischen Voraussetzungen (Jura, Voralpen, Alpen) eine wichtige Ergänzung unserer methodischen Erfahrungen. Die Ergebnisse werden 1984 publiziert. Neben dem praktischen Ziel der Inventarisierung dürften diese Ergebnisse des IVS eine wichtige Grundlage für weitere wissenschaftliche Arbeiten außerhalb des Inventars bilden.

Der offizielle Start des IVS erfolgte am 1.1.1984. Aufgrund der vorgenommenen Zeitberechnungen sollte es möglich sein, die Inventarisierungsarbeiten nach vorliegendem Konzept im Zeitraum von 12 Jahren bis 1995 abzuschließen.

\section{Bibliographie}

AERNI, K. (1961): Die alten Paßwege Albrun, Grimsel, Gries, Mt. Moro und Loetschen. Bern.

AERNI, K. (1971): Die Paßwege Gemmi, Loetschen, Grimsel. Bern.

AERNI, K. (1975): Gemmi - Loetschen - Grimsel. In: Jahrbuch der Geogr. Gesellschaft Bern, Band 51/1973-74, 23-61.
AERNI, K. und PFISTER, C. (1973): Der Kulturlandschaftswandel im Moosseeraum. Jahresbericht der Geogr. Gesellschaft Bern 50/1970-1972, S. 185-235.

BARRAUD, Ch. und HERZIG, H. (1983): Altstraßenforschung in der Schweiz. In: Archäologie der Schweiz, 6/1983: S. 137-141, Basel.

BLN (1977): Bundesinventar der Landschaften und Naturdenkmäler von nationaler Bedeutung. Bern.

EJP (1981): Erläuterungen zum Bundesgesetz über die Raumplanung, S. 230.

EWALD, K. (1978): Der Landschaftswandel. Zur Veränderung schweizerischer Kulturlandschaften im 20.Jahrhundert. Birmensdorf

GEBESSLER, A. und EBERL, W. (1980): Schutz und Pflege von Baudenkmälern in der BRD. Stuttgart.

GROSJEAN, G. (1982): Raumtypisierung nach geographischen Gesichtspunkten als Grundlage der Raumplanung auf höherer Stufe. Geographica Bernensia P1, Bern, 3. Auflage.

ISOS (1976): Ortsbild - Inventarisation. Aber wie? Eidg. Oberforstinspektorat, Zürich.

IVS (1983): Bibliographie IVS 1982: Inventar historischer Verkehrswege der Schweiz (IVS).

id.: Einzelne Kantone

KLN (1979): Inventar der zu erhaltenden Landschaften und Naturdenkmäler von nationaler Bedeutung. Basel (3. rev. Ausg.).

MÜLLER, J. (1973): Alle Jahre wieder saust der Preßlufthammer nieder oder die Veränderung der Landschaft. Aarau.

NHG (1966): Bundesgesetz über den Natur- und Heimatschutz.

OFI: Geschäftsberichte des Oberforstinspektorates 1975-1980. Bern.

PFISTER, C. (1977): Autobahnen verändern eine Landschaft. Geographica Bernensia S 2, Bern.

PROBST, R. (1981): Der Wägesse - Ein historischer Verkehrsweg. Geogr. Inst. Bern.

RPG (1979): Bundesgesetz über die Raumplanung.

RUCKLI, R. (1960): Nationalstraßenbau und Landschaftsschutz. In: Straße und Verkehr 1960, Bd.1, Zürich, S. 3-7.

SCHWARZE, M. (1983): Die Erhaltung traditioneller Kulturlandschaften dokumentiert an einigen Fallbeispielen in verschiedenen Landschaftsteilen der Schweiz. Zürich.

WEISS, H. (1981): Die friedliche Zerstörung der Landschaft und Ansätze zu ihrer Rettung in der Schweiz. Zürich. 